\title{
Phase-Correlated Nondirectional Laser Emission from the End Facets of a ZnO Nanowire
}

\author{
Lambert K. van Vugt, ${ }^{*} \ddagger$ Sven Ruhle, ${ }^{, \neq \ddagger}$ and Daniël Vanmaekelbergh \\ Condensed Matter and Interfaces, Debye Institute, Utrecht University, \\ P.O. Box 80 000, 3508 TA Utrecht, The Netherlands.
}

Received July 14, 2006; Revised Manuscript Received September 21, 2006

\begin{abstract}
We investigated the laser emission from individual $\mathrm{ZnO}$ nanowires and observed an interference pattern due to coherent laser emission from the wire end facets. Comparison with numerical simulations shows that the laser light is emitted nearly spherically from the wire ends. The energy spacing between sharp lasing modes scales with the inverse length of the nanowire; thus, laser emission peaks correspond to FabryPérot modes of the nanowire cavity.
\end{abstract}

\section{Introduction}

Semiconductor nanowires show extraordinary optical properties such as wave guiding ${ }^{1-3}$ and lasing ${ }^{4-10}$ and, thus, form a class of very promising building blocks for novel miniaturized optical and optoelectronic devices. ${ }^{11,12}$ Nanowires are currently among the smallest known lasing devices, with lengths between 1 and $50 \mu \mathrm{m}$ and diameters that can be significantly smaller than the emission wavelength in vacuum. In contrast to microcavities with distributed Bragg reflectors, ${ }^{13}$ nanowires form an optical cavity due to the refractive index difference with its surroundings.,10,14-16 At high excitation intensities, sharp laser peaks appear in the luminescence spectrum, and a highly nonlinear input-output characteristic has been observed from $\mathrm{GaN},{ }^{4} \mathrm{ZnO},{ }^{5} \mathrm{CdS},{ }^{6}$ $\mathrm{ZnS},{ }^{7}$ and $\mathrm{GaSb}^{8}$ nanowires. Such nanolasers are usually pumped optically, but electrically driven lasers have also been demonstrated. ${ }^{9}$ The development of coherent light sources on the nanoscale opens the door toward miniaturized spectroscopic systems and photonic circuits. On the other hand, extended research is required to investigate novel effects due to the small diameter of the nanolaser cavity, ${ }^{17}$ which is often beyond the diffraction limit of the emitted light. We observe laser emission from the nanowire end facets with diameters ranging from 200 to $400 \mathrm{~nm}$, but the lasing of thinner wires is also known from the literature. ${ }^{10}$ While, in macroscopic laser systems, the emission is highly directional, it is not obvious how the light is emitted from facets with subwavelength dimensions. Theoretical investigation of the far field predicts that the angular emission intensity should depend strongly on the mode type (transverse

\footnotetext{
* Corresponding authors. E-mail: L.K.vanVugt@phys.uu.nl; S.Ruhle@ phys.uu.nl. Fax +31 (0)30 2532403.

$\doteqdot$ These authors contributed equally to the presented work.
}

electric (TE), transverse magnetic (TM), or hybrid (HE, EH) modes) and could be spread over a large emission angle. ${ }^{18}$

Here we report experimental evidence for highly nondirectional emission from lasing $\mathrm{ZnO}$ nanowires. We observe a clear interference pattern of two emission sources located at the two nanowire ends. A detailed analysis reveals that the interference pattern depends not only on the emission wavelength and the wire length but also on the optical components of the measurement instrument (microscope). ${ }^{19}$ This enabled us to simulate interference patterns for different angular emission distributions from the wire ends. Comparison with experimentally measured patterns shows that a good agreement with the simulations can only be achieved if the two sources have a fixed phase relation and light waves emitted from the end facets have strong components in the side- and backward directions, that is, the ends act nearly as point sources. Furthermore, we show that the laser emission peaks correlate well with Fabry-Pérot (FP) modes of the nanowire cavity.

$\mathrm{ZnO}$ nanowires with diameters ranging from 60 to 400 $\mathrm{nm}$ and lengths of up to $20 \mu \mathrm{m}$ were grown vertically on oriented sapphire substrates using the carbothermal reduction method. ${ }^{20}$ Details of the synthesis can be found elsewhere. ${ }^{21}$ After growth, the wires were mechanically broken off and dispersed onto $\mathrm{SiO}_{2}$-covered (500 nm) Si substrates, on which gold markers had been defined by electron beam lithography. This enabled us to locate individual wires and to perform optical measurements and scanning electron microscopy (SEM) on the same nanowires. Optical experiments were performed using a pulsed Nd:YLF laser (10 ns pulse length) at $349 \mathrm{~nm}$ with a tunable repetition rate of $2-5 \mathrm{kHz}$, which was weakly focused onto the nanowires using an optical microscope (Zeiss Axioplan 2) with an infinity-corrected objective $(100 \times$ magnification, NA 0.9$)$. The spot diameter 


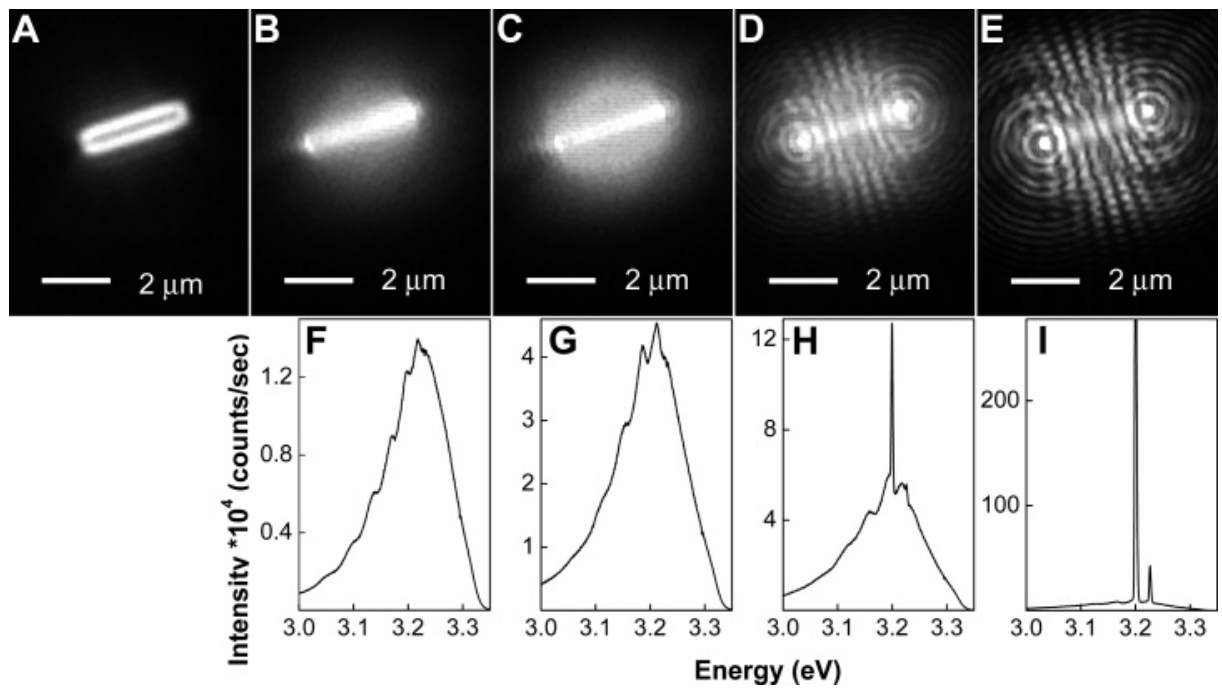

Figure 1. (A) Dark-field optical image of a $\mathrm{ZnO}$ nanowire laser. (B-E) Panchromatic PL images of the nanowire with increasing excitation intensity: (B) $24 \mathrm{~W} / \mathrm{cm}^{2}$, (C) $93 \mathrm{~W} / \mathrm{cm}^{2}$, (D) $139 \mathrm{~W} / \mathrm{cm}^{2}$, and (E) $268 \mathrm{~W} / \mathrm{cm}^{2}$. (F-I) PL spectra taken at the excitation intensities corresponding to images $\mathrm{B}-\mathrm{E}$.

of the laser excitation, approximately $20 \mu \mathrm{m}$, was such that the entire volume of the wires was excited while the excitation power was adjusted using different combinations of neutral density filters. The emission was collected by the same objective, and the excitation wavelength was filtered by a dichroic mirror and a long pass filter. The emission was either projected onto a charge-coupled device (CCD) camera for imaging or coupled by a multimode fiber into an Acton Research 300spi $0.3 \mathrm{~m}$ spectrometer, fitted with a liquid nitrogen-cooled Princeton research CCD for spectral analysis.

An optical dark field image of a $3.2 \mu \mathrm{m}$-long $\mathrm{ZnO}$ nanowire is shown in Figure 1A. The panchromatic photoluminescence (PL) image of the same wire is shown in Figure $1 \mathrm{~B}-\mathrm{E}$ under uniform illumination with an expanded beam spot from the Nd:YLF laser. The excitation power was increased stepwise from below the lasing threshold until strong laser emission was observed. The accompanying emission spectra are shown in Figure 1F-I. The UV spectrum below the lasing threshold (Figure 1F) of this wire is broad and shows small modulations due to FP-type interference as a result of multiple reflections between the wire end facets. As soon as the excitation power exceeds the lasing threshold $\left(100 \mathrm{~W} / \mathrm{cm}^{2}\right.$ for this wire), sharp peaks appear in the spectrum (Figure $1 \mathrm{H}$ ), and rings around the nanowire end facets are observed in the CCD image. Similar, but more diffuse patterns arising above the lasing threshold have been reported before. ${ }^{10,22}$ At the higher excitation intensities, the emitted spectrum consists mainly of laser emission with clearly distinguishable peaks (Figure 1I). Depending on the batch, we observed that $10-50 \%$ of the wires show lasing, while the interference pattern depends on the emission wavelength and the wire length. For most wires, we observed more than one lasing peak with a peak spacing that correlates well with the reciprocal wire length $\left(L^{-1}\right)$ :

$$
\Delta \omega=\pi c /(n L)
$$

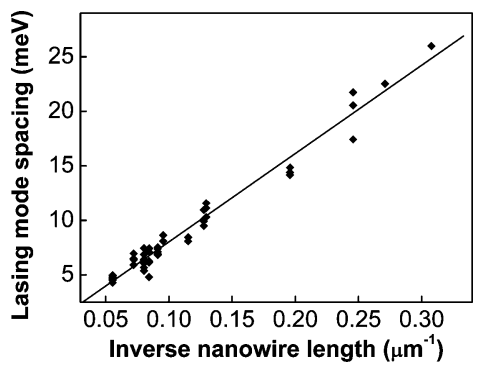

Figure 2. Mode spacing of the lasing peaks as a function of reciprocal nanowire length.

with the speed of light, $c$, the refractive index, $n$, and the nanowire length, $L$. A plot of the mode spacing $\Delta \omega$ versus reciprocal wire length is shown in Figure 2 (in units of meV) for several samples. Good quantitative agreement of the data points with a linear least-square fit strongly indicates that the lasing peaks are due to FP eigenmodes with an integer number of $\lambda / 2$ that fits into the length direction of the nanowire. The spread in mode spacing from each wire (vertical group of points at a given inverse wire length) indicates that the refractive index is not constant in the emission range. The FP modes observed in the luminescence spectrum below the lasing threshold (Figure 1F) and the lasing FP modes show that the nanowire forms an optical cavity, caused by the refractive index difference between and the wire and the surrounding. We remark that the substrate did not have a significant influence on the laser emission; lasing was also observed when the $\mathrm{SiO}_{2}$-covered $\mathrm{Si}$ substrate was replaced by sapphire, graphite, or gold substrates. This indicates that the electromagnetic coupling to the substrate is rather weak.

To investigate the directionality of the laser emission, we compared the experimentally observed diffraction patterns with simulations, calculated for different angular emission profiles from the wire end facets. Figure $3 \mathrm{~A}$ shows the experimental geometry with the nanowire located in the focal plane of an infinity-corrected objective in conjunction with 

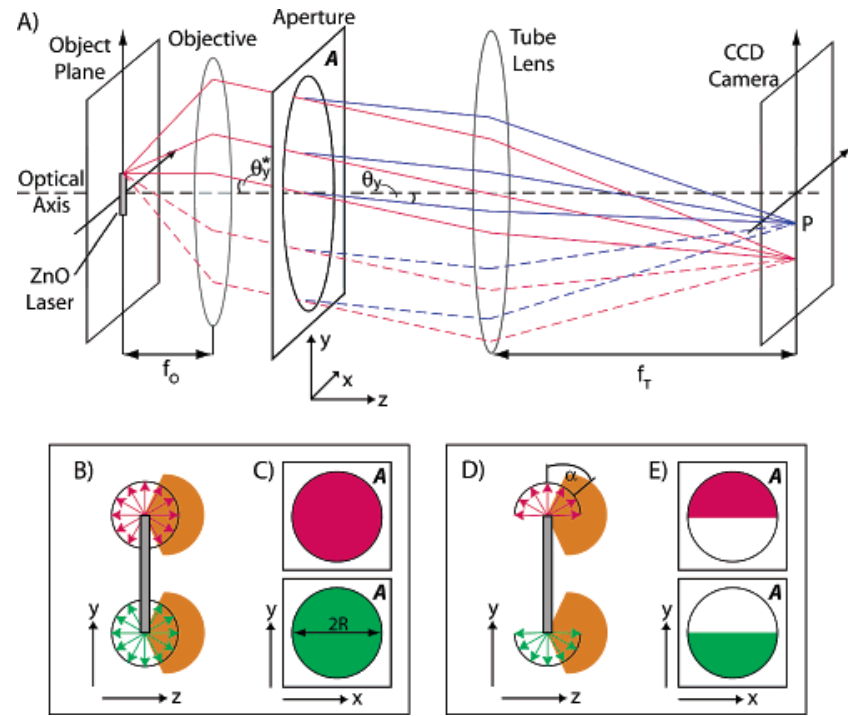

Figure 3. (A) Experimental configuration. For simplicity, only light emission from the upper end facet of the $\mathrm{ZnO}$ nanowire laser is shown. Red solid lines indicate light rays for hemispherical emission, while solid and dashed lines together indicate spherical emission. Blue rays show the formation of the interference pattern. (B) Schematic spherical laser emission from the upper and lower end facet, depicted as red and green arrows, respectively. (C) Intensity distribution in the aperture, originating from the emission of the upper and lower end facet. (D) The same as B for hemispherical emission. (E) Hemispherical emission from the upper facet leads to an intensity contribution in the upper half of the aperture (depicted in red, see top panel), while emission from the lower end facet gives a constant light intensity in the lower aperture half (depicted in green, see bottom).

a tube lens. Light emitted from the nanowire in the object plane is collimated by the objective and projected by the tube lens onto a CCD camera. This configuration is widely used in modern microscopes since it allows easy insertion of optical elements such as polarizers, filters, and so forth into the parallel light beam between the objective and the tube lens without further optical corrections. The magnification $M$ is determined by $M=f_{\mathrm{O}} / f_{\mathrm{T}}$, where $f_{\mathrm{O}}$ and $f_{\mathrm{T}}$ are the focal lengths of the objective and tube lens, respectively. Figure $3 \mathrm{~A}$ shows that the beam path inside the microscope is equivalent to that of an experimental configuration to measure Fraunhofer diffraction, ${ }^{19}$ where the diffraction pattern of an incident parallel light beam is projected onto a CCD array by the tube lens. The illuminated part of the aperture can be considered as an ensemble of elementary oscillators, re-emitting the light originating from the nanowire end facet. Due to diffraction, regions of constructive and destructive interference occur, which are observed as bright and dark regions on the CCD camera behind the tube lens. Hence, the electric field $E$ at a point $P$ on the CCD array is proportional to the integral of the fields originating from all elementary oscillators within the aperture area $A$, multiplied with their phase,

$$
E_{\mathrm{P}} \propto \iint_{A} f(x, y) e^{\left(-i k_{0}\left(\left(l^{*}+l\right) x+\left(m^{*}+m\right) y\right)\right)} \mathrm{d} x \mathrm{~d} y
$$

The coordinates of $P$ are defined by $l=\sin \theta_{x}$ and $m=$ $\sin \theta_{y}$, where $\theta_{x}=90^{\circ}-\varphi_{x}$ and $\theta_{y}=90^{\circ}-\varphi_{y}$ are defined by the angles $\varphi_{x}$ and $\varphi_{y}$ between the beam path and the $x$ and $y$-coordinate axes, respectively. The angles of the incident parallel light beam with respect to the aperture plane are defined by $l^{*}=\sin \theta_{x}^{*}$ and $m^{*}=\sin \theta_{y}^{*}$. Figure $3 \mathrm{~A}$ shows the experimental geometry for a nanowire in the object plane, aligned parallel to the $y$-axis. The function $f(x, y)$ describes the intensity distribution across the aperture and contains information about the direction of the laser emission from the nanowire. Figure 3B,D shows a magnification of a nanowire lying in the focal plane of the microscope. To investigate the spatial laser emission from the wire ends, we compared the uniform emission in all directions (spherical emission, Figure 3B) with the case in which light is only emitted in the forward direction such that the intensity is constant for emission angles $|\alpha| \leq 90^{\circ}$ and zero for $|\alpha|>$ $90^{\circ}$ (hemispherical emission, Figure 3D). The orange background in Figure 3B,D indicates the light collection cone of the microscope objective, defined by its numerical aperture, NA (0.9 in air), which corresponds to a collection angle of $128^{\circ}$. For nondirectional (i.e., spherical) emission, the aperture is completely illuminated (see solid and dashed light rays in Figure 3A); hence, we use an intensity distribution $f(x, y)=1$ for $x^{2}+y^{2} \leq R^{2}$ (with the origin in the center of the aperture of radius $R$ ). Figure $3 \mathrm{C}$ shows the uniform contributions coming from the upper (red) and lower (green) end facet of the nanowire. We note that the phase factor $e^{\left(-i k_{0}\left(l^{\prime} x+m^{\prime} y\right)\right)}$ is different for the emission from the top and bottom facets, thus leading to different contributions in the diffraction pattern. For a nanowire with directional (i.e., hemispherical) emission, the top end facet illuminates the upper half of the aperture (see solid light rays in Figure 3A) such that $f(x, y)=1$ for $x^{2}+y^{2} \leq R^{2}$ and $y \geq 0$ (see red area in Figure 3E, top). The bottom end facet illuminates the lower half of the aperture: $f(x, y)=1$ for $x^{2}+y^{2} \leq R^{2}$ and $y \leq 0$ (see green area in Figure 3E, bottom). The different diffraction integrals (eq 2) for spherical and hemispherical emission lead to distinct interference patterns and thus allow a clear distinction between directional (hemispherical) and nondirectional (spherical) emission from the end facets (see below).

Experimental steady-state diffraction patterns of a long $(10.2 \mu \mathrm{m})$ and short $(2.1 \mu \mathrm{m})$ nanowire are presented in Figure $4 \mathrm{~A}, \mathrm{~B}$, and the respective spectra of these wires are shown in the insets. The CCD image of the long nanowire shows characteristic similarities with a diffraction pattern of a round aperture generated by two distinct sources at the nanowire end facets, which are sufficiently far apart such that hardly any interference is observed. The spacing between the minima is determined by the nanolaser wavelength and the radius of the aperture. Furthermore, one can see weak luminescence from the nanowire itself due to incoherent emission. The short wire (Figure 4B), in contrast, shows strong interference between the two emission sources, leading to a ray-like pattern due to the formation of interference maxima and minima.

Simulated patterns for nondirectional (spherical) emission are shown in Figure 4C,D. For the long wire, one can see that the ring pattern around the nanowire ends is nicely reproduced in this simulation. Furthermore, one can see in 

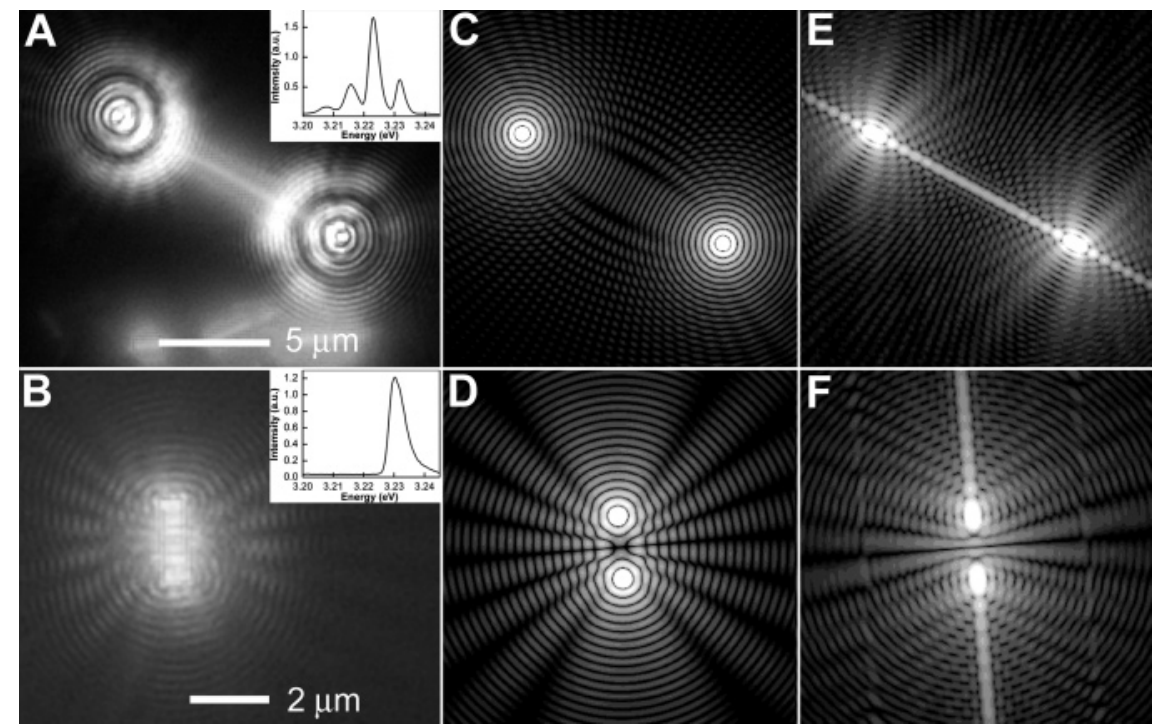

Figure 4. Left: Experimentally observed diffraction pattern of a $10.2 \mu \mathrm{m}$ long nanowire showing no interference (A) and a $2.1 \mu \mathrm{m}$ short nanowire with strong interference (B). Middle: Simulated diffraction pattern for the long (C) and short (D) nanowire using spherical emission from the wire end facets. Right: Simulated diffraction pattern for the long (E) and short (F) wire based on hemispherical emission.

the simulations some weak interference between the wire ends, which is not observed in the CCD image, probably due to an insufficient sensitivity of the CCD camera. We note that the simulations do not contain incoherent luminescence so that the nanowire itself is not visible in the simulations. The calculated pattern for the short wire (Figure 4D) shows the same type of interference minima between the two lasing sources, which originate from a superposition of the emitted light from both ends with a fixed phase difference. Full destructive interference can only be observed if the phase difference between the two emission sources is zero or an integer multiple of $\pi$. The measured CCD image thus provides direct experimental evidence for a zero or fixed phase shift between the emission sources. Calculated diffraction patterns for hemispherical emission are shown in Figure 4E,F. Neither the ring structure around the lasing sources (Figure 4E) nor the interference pattern (Figure 4F) of the measurements is reproduced correctly. For both wires, one observes a pronounced interference pattern along the wire axis, exceeding the wire length, in contrast to that found experimentally. Furthermore, one can see that, for the short wire, the number of interference minima differs in experiment and simulation. We conclude that lasing emission from the end-facets must occur in a nearly spherical manner.

Observed intensity modulations in the ring patterns at the wire ends (Figure 4A), are not present in the simulations (Figure 4C). This small difference might be caused either by beating of the different emission wavelengths, or by a nondirectional emission distribution slightly different from the assumed spherical emission. For the short wire, the measured interference pattern around the end facets (Figure 4B) differs slightly from the calculated one (Figure 4D); this might be due to the finite wire width, which was also not included in the simulations (emission from the end facets was approximated by point sources). The overall good agreement between experiments and simulations based on spherical emission brings us to the conclusion that spherical emission from $\mathrm{ZnO}$ nanowires in the investigated diameter range is a good approximation of the real angular distributed emission. This is in good agreement with theoretical work on the far field of lasing nanowires, which predicts that the $\mathrm{TM}_{01}$ and $\mathrm{TE}_{01}$ modes can emit light in a wide angular range. ${ }^{18} \mathrm{We}$ furthermore mention that laser light from $\mathrm{HE}_{11}$ modes is predicted to be mainly emitted in the direction of the nanowire length axis, and thus might not be detectable in our experimental configuration, where the nanowire is oriented perpendicular to the optical axis of the microscope (see Figure 3A).

For novel applications based on nanowire lasers with subwavelength diameter, the nondirectional emission has to be taken into consideration. While this effect might be beneficial for devices such as flat panel displays, where a wide emission angle guaranties good visibility from different observation angles, it might be a drawback for nanosized photonic devices where a well-defined emission direction is required.

In conclusion, we have shown that interference and diffraction from individual $\mathrm{ZnO}$ nanolasers has been observed. Numerical simulations show that good agreement with experiments can be achieved only if nondirectional emission from the end facets is assumed. Furthermore, we have demonstrated that the energy spacing between neighboring lasing peaks corresponds well with FP modes.

Acknowledgment. This work is part of the research program of the "Stichting voor Fundamenteel Onderzoek der Materie (FOM)", which is financially supported by the Nederlandse Organisatie voor Wetenschappelijk Onderzoek (NWO). This work is supported by NanoNed, a national nanotechnology program coordinated by the Dutch Ministry of Economic Affairs. 


\section{References}

(1) Law, M.; Sirbuly, D. J.; Johnson, J. C.; Goldberger, J.; Saykally, R. J.; Yang, P. Science 2004, 305, 1269.

(2) Pan, A.; Liu, R.; Yang, Q.; Zhu, Y.; Yang, G.; Zou, B.; Chen, K. J. Phys. Chem. B 2005, 109, 24268.

(3) Tong, L.; Gattass, R. R.; Ashcom, J. B.; He, S.; Lou, J.; Shen, M.; Maxwell, I.; Mazur, E. Nature 2003, 426, 816.

(4) Gradecak, S.; Qian, F.; Li, Y.; Park, H. G.; Lieber, C. M. Appl. Phys. Lett. 2005, 87, 173111

(5) Huang, M. H.; Mao, S.; Feick, H.; Yan, H.; Wu, Y.; Kind, H.; Weber, E.; Russo, R.; Yang, P. Science 2001, 292, 1897.

(6) Agarwal, R.; Barrelet, C. J.; Lieber, C. M. Nano Lett. 2005, 5 (5), 917.

(7) Ding, J. X.; Zapien, J. A.; Chen, W. W.; Lifshitz, Y.; Lee, S. T.; Meng, X. M. Appl. Phys. Lett. 2004, 85 (12), 2361.

(8) Chin, A. H.; Vaddiraju, S.; Maslov, A. V.; Ning, C. Z.; Sunkara, M. K.; Meyyappan, M. Appl. Phys. Lett. 2006, 88, 163115.

(9) Duan, X.; Huang, Y.; Agarwal, R.; Lieber, C. M. Nature 2003, 421, 241.

(10) Johnson, J. C.; Yan, H.; Yang, P.; Saykally, R. J. J. Phys. Chem. B 2003, 107, 8816.

(11) Pettersson, H.; Trägardh, J.; Persson, A. I.; Landin, L.; Hessman, D.; Samuelson, L. Nano Lett. 2006, 6 (2), 229.
(12) Huang, Y.; Duan, X.; Lieber, C. M. Small 2005, 1 (1), 142.

(13) Bayer, M.; Forchel, A.; Reinecke, T. L.; Knipp, P. A.; Rudin, S. Phys. Status Solidi A 2002, 191 (1), 3.

(14) Wang, D.; Seo, H. W.; Tin, C. C.; Bozack, M. J.; Williams, J. R.; Park, M.; Tzeng, Y. J. Appl. Phys. 2006, 99, 093112.

(15) Nobis, T.; Kaidashev, M.; Rahm, A.; Lorenz, M.; Grundmann, M. Phys. Rev. Lett. 2004, 93 (10), 103903.

(16) Hauschild, R.; Lange, H.; Priller, H.; Klingshirn, C.; Kling, R.; Waag, A.; Fan, H. J.; Zacharias, M.; Kalt, H. Phys. Status Solidi B 2006, $243(4), 853$

(17) Maslov, A. V.; Bakunov, M. I.; Ning, C. Z. J. Appl. Phys. 2006, 99 , 024314.

(18) Maslov, A. V.; Ning, C. Z. Opt. Lett. 2004, 29 (6), 572.

(19) Born, M. Optik; Springer-Verlag: Berlin, 1933.

(20) Huang, M. H.; Wu, Y.; Feick, H.; Tran, N.; Weber, E.; Yang, P. Adv. Materials 2001, 13 (2), 113.

(21) Prasanth, R.; van Vugt, L. K.; Vanmaekelbergh, D. A. M.; Gerritsen, H. C. Appl. Phys. Lett. 2006, 88, 181501.

(22) Sirbuly, D. J.; Law, M.; Yan, H.; Yang, P. J. Phys. Chem. B 2005, 109, 15190.

NL0616227 